\title{
Using Multivariate Quantitative Genetics Theory to Assist in EA Customization
}

\author{
Jeffrey K. Bassett \\ George Mason University \\ 4400 University Dr, MS 4A1 \\ Fairfax, Virginia \\ jbassett@cs.gmu.edu
}

\author{
Kenneth A. De Jong \\ George Mason University \\ 4400 University Dr, MS 3A5 \\ Fairfax, Virginia \\ kdejong@gmu.edu
}

\begin{abstract}
Customizing and evolutionary algorithm (EA) for a new or unusual problem can seem relatively simple as long as one can devise an appropriate representation and reproductive operators to modify it. Unfortunately getting a customized EA to produce high quality results in a reasonable amount of time can be quite challenging. There is little guidance available to help practitioners deal with this issue. Most evolutionary computation (EC) theory is only applicable to specific representations, or assumes knowledge of the fitness function, such as the location of optima.
\end{abstract}

We are developing an approach based on theory from the biology community to address this problem. Multivariate quantitative genetics theory characterizes evolving populations as multivariate probability distributions of phenotypic traits. Some advantages it offers are a degree of independence from the underlying representation, and useful concepts such as phenotypic heritability. Re-working the quantitative genetics equations, we expose an additional term that we call "perturbation". We believe that perturbation and heritability provide quantitative measures of the exploration and exploitation, and that practitioners can use these to identify and diagnose imbalances in customized reproductive operators.

To illustrate, we use these tools to diagnose problems with a standard recombination operator for a Pittsburgh approach classifier system. With this knowledge we develop a new, more balanced, recombination operator, and show that its use leads to significantly better results.

\section{Categories and Subject Descriptors}

F.2 [Theory of Computation]: Analysis of Algorithms and Problem Complexity; G.3 [Mathematics of Computing]: Probability and Statistics-Correlation and regression analysis, Multivariate statistics

\section{General Terms}

Theory, Algorithms, Performance

\section{Keywords}

Evolutionary Computation, Quantitative Genetics, Heritability, Customization

\section{INTRODUCTION}

When using an EA to solve a new or unusual problem, two general strategies are available. The first is to encode the problem in a traditional linear representation and use the standard reproductive operators. Unfortunately this encoding process can often transform the fitness landscape to one that can be much more difficult to search. In some cases such an encoding may not even be possible, especially if potential solutions can differ in size.

The second approach is to customize the EA for the specific problem. This means using a representation that more naturally describes a potential solution to the problem, and then developing reproductive operators that can modify it. The problem with this approach is that it is not always obvious how to proceed, and sometimes it can be quite difficult to build operators that allow the EA to find high fitness solutions in a reasonable amount of time.

Ideally one would turn to theory for advice on performing customizations. Unfortunately EC theory tends not to be applicable in these situations. Most theory is either specific to certain representations, or requires detailed knowledge about the fitness landscape being searched. The practitioner is left to using an ad-hoc approach to perform their customizations. Our goal is to help provide a more principled approach.

We propose using multivariate quantitative genetics theory from the biology community to address this issue. This theory has the advantage of offering a degree of representation independence, which it achieves by dealing with individuals using only quantitative (e.g. real-valued) phenotypic traits. Populations are characterized as multivariate probability distributions of these traits, and a series of equations are used to model how these distributions change from one population to the next as a result of selection and reproduction. As part of these equations, the theory defines useful concepts like heritability that describe mathematically how 
well traits are retained during reproduction. We believe this provides a measure of a reproductive operator's contribution to an algorithm's ability to exploit good solutions.

Multivariate heritability also suggests a way of identifying where problems in a reproductive operator may lie. This can be determined by comparing the population's traits distributions before and after an operation is performed, to see how similar they are. When dissimilarities are found we can identify which sets of traits are most affected. If the traits have been carefully chosen, it will be possible trace the affected traits back to associated changes in the genome. Once we learn what is causing the distributions to differ, we can reason about how to improve the operators or representation in a way that will reduce or resolve the problem. Assuming one has chosen a set of traits that reasonably represent the search space, it should be possible to discover most problems with one's customized algorithm using this approach.

To illustrate the value of this theory, we will demonstrate its use in diagnosing a problem with a specific recombination operator. In previous work of ours using Pittsburgh approach classifiers systems, we noticed that the customized recombination operators often produced worse results than using standard EA operators on fixed length strings. This makes them a good candidate for attempts at improvement. We demonstrate that the two-point Pittsburgh recombination has lower heritability than the standard two-point recombination operator. We also show how problems with the operators can be identified by examining the way the operators transform the trait distributions of the populations. Using the knowledge we gain from this, we then develop a recombination operator that has much higher heritability, and correspondingly finds better solutions to a function approximation problem.

Multivariate quantitative genetics can act as the framework for a more principled approach to customizing EAs for new problems. As long as one can identify an appropriate set of traits to measure, the theory offers a representation independent way of modeling evolutionary processes and identifying problems within.

\section{BACKGROUND}

We offer a brief review of quantitative genetics, focusing particularly on those areas that pertain to our work. We will also review two areas of EC research that have, at least at times, applied quantitative genetics theory: evolvability theory and estimation of distribution algorithms (EDAs).

\subsection{Quantitative Genetics}

Quantitative genetics [6, 20] is concerned with measurable phenotypic traits that are statistically modeled at a population level. Statistical measures like mean, variance and covariance are used to characterize populations and the relationships between them. Several equations are then used to model the effects of selection, reproduction and genetic drift over time.

Quantitative genetics equations are often decomposed into meaningful terms and factors, each of which represents some important aspect of the evolutionary process. For example, Price's theorem [19] separates the average effects of selec- tion and reproduction into two terms. Similarly, the equation for population variance includes terms for the effects of heritability, epistasis and variation due to the environment. Decompositions like these have the potential to offer insights into how and why certain operators and representations are not performing well.

Perhaps the most notable of these equations is the breeder's equation [6] which models the response to selection,

$$
R=h^{2} S
$$

Here $S$ represents the selection differential, $h^{2}$ is heritability and $R$ is the response to selection. In very simplistic terms, $S$ describes the change in the average value of a trait caused by selection culling low fitness individuals from the population. This description is not completely accurate though since high fitness individuals that are selected multiple times to be parents are counted as if multiple copies of them were in the population as well.

The response to selection $(R)$ describes the change in the trait's average within the population from one generation to the next. Heritability $\left(h^{2}\right)$ is a statistical measure of similarity between the selected parents and their offspring. It can also be thought of as indicating how well a trait is transmitted from a parent to its offspring during reproduction [2]. In this light, the breeder's equation can be read as follows: If selection causes an increase or decrease in the mean value of a trait, then the closer heritability is to 1 , the more that change will also be manifested in the next generation.

Heritability is often estimated as a regression coefficient between parent and offspring trait values using the following equation,

$$
h^{2}=\tau \frac{\operatorname{cov}\left(o_{i}, p_{i}\right)}{\operatorname{var}\left(p_{i}\right)},
$$

where $\tau$ is the number of parents per offspring ( 1 for asexual reproduction and 2 for sexual reproduction), $o_{i}$ is the value of the phenotypic trait for offspring $i$, and $p_{i}$ is the trait values of the parents of $i$ averaged together (also known as the midparent).

Values for $h^{2}$ tend to fall in the range 0 to 1 , but are not limited to this. Note that, in the literature, the term for heritability is always $h^{2}$ and not $h$ for historical reasons, even though $h$ has little meaning by itself.

\subsection{Evolvability}

There are several cases where quantitative genetics theory has already been applied to EAs. One of the first was research done by Altenberg [1] in which he used Price's theorem [19] as the foundation for an infinite population dynamical systems model of EAs. His main result was to re-derive the schema theorem and show that recombination was essential for the schema theory to hold. His work also provided a theoretical justification for using the covariance between parent and offspring fitness as a predictor of EA performance, which up until then had been just a heuristic. Interestingly Altenberg might have found it easier to derive this result from the variance equations rather than Price's theorem, which measures changes in population means. Asoh 
and Mühlenbein [3] did just this when they examined heritability in EAs.

The importance of the relationship between parent and offspring fitness was known in the EA community before Altenberg's and Mühlenbein's work, and is one of the most effective tools for assisting the customization process. Using this measure, one can compare two operators to see which is more likely to improve an EAs performance [13]. Unfortunately it offers no indication of why an operator is performing poorly, and no suggestions for how to improve it.

Parent-offspring fitness covariance is also used as a measure of fitness landscape difficulty [23, 22, 9]. For example, consider a landscape where the fitness of neighboring points have no relationship to one-another. Without near-perfect knowledge of the landscape, no set of operators could perform well on this problem. On the other hand a poor choice of reproductive operators can have a similar effect by searching the landscape in an irregular and unpredictable way. Unfortunately, it is difficult to tell which case one is faced with.

Some have built tools based on quantitative genetics for evaluating the components of an EA either before or during a run. Langdon [12] used both Price's theorem and Fisher's fundamental theorem [18] to build his tools, but these make certain assumptions about the structure of the genome which make them somewhat representation dependent. Potter, Bassett and De Jong [17] also explored building tools with Price's theorem. Their results demonstrated the importance of reproductive variance in analyzing operators, leading them to explore approaches for measuring variance using Price's theorem as well [4] [5].

\subsection{Estimation of Distribution Algorithms}

Mühlenbein and Schlierkamp-Voosen [16] used the breeder's equation [6] to guide the design of their Breeder Genetic Algorithm (BGA). An analysis of crossover using this same equation led to the development of gene-pool crossover operators and then to the development of Estimation of Distribution Algorithms (EDAs) [15, 14]. EDAs differ from EAs in that they do not use standard reproductive operators. In each generation they estimate the probability distributions of the gene frequencies in the selected parents and use this information to generate a new population of offspring. Quantitative genetics played a continuing role in Mühlenbein's work, guiding the development of several new EDAs.

A newer branch of EDA research, called continuous EDAs [24, 25], takes an approach that is more like modeling phenotypic traits. As the name implies, an individual is represented by a set of real valued traits. This allows population distributions to be modeled using joint Gaussian distributions instead of just tracking genes values. The use of covariance matrices also allows epistatic relationships to be captured by orienting the distribution along a diagonal. The disadvantage to this approach is that it limits the types of genetic structures that can be modeled, only being applicable to real-valued optimization problems.

Statistical approaches such as quantitative genetics have played an important role in the process of customizing EAs.
In particular measures similar to heritability of fitness have been some of the most effective tools to date for aiding adhoc design approaches. Next we will discuss Multivariate Quantitative Genetics and how it can assist in actually diagnosing problems.

\section{MULTIVARIATE QUANTITATIVE GENET- ICS}

So far, the quantitative genetics equations we have examined have dealt with only a single phenotypic trait. To observe how the population behaves in the search space as a whole, we need to consider multiple traits simultaneously. An extension to this theory called multivariate quantitative genetics [10, 11] was developed to address these kinds of issues. The equations essentially mirror the standard equations, but they use vectors and matrices to simultaneously manage several traits, and the interactions between them. For example, here is the multivariate version of equation 1, the breeder's equation:

$$
\Delta \bar{z}=\mathbf{G P}^{-1} \mathbf{S}
$$

The individuals $o_{i}$ and $p_{i}$ are now described by a group of traits and are represented as vectors, as is the selection differential $(\mathbf{S})$ and the response to selection $(\Delta \bar{z})$. $\mathbf{G}$ is the cross-covariance matrix defined by $\tau \operatorname{cov}\left(o_{i}, p_{i}\right)$ and $\mathbf{P}$ is the covariance matrix describing the distribution of traits of the selected parents $\left(\operatorname{var}\left(p_{i}\right)\right)$. Heritability is now defined by the matrix $\mathbf{G P}^{-1}$, and high heritability values will be those near the identity matrix.

Biologists tend not to think in terms of heritability when using the multivariate form of the breeder's equation though. Instead they have worked under the assumption that the $\mathbf{G}$ and $\mathbf{P}$ matrices will remain stable over time, especially in the short term. If this is true, it is not difficult to see that heritability will remain constant, and therefore the breeder's equation could still be used to do prediction.

Our concerns are somewhat different from those of the biologists. Instead of predicting the algorithms behavior, we want to diagnose problems with the reproductive operators.

\subsection{Modified Equations}

We believe that heritability is an important metric to consider when developing a new operator, but it is clearly not the only important factor. For example, an operator that just produces clones of the parents is guaranteed to have the highest heritability possible, and yet we would not expect it to produce particularly good results.

We have examined the quantitative genetics equations again from first principles to see if we could identify other factors that could assist us during the design process. One common assumption we find is that all individuals will have the same number of parents. This makes it difficult to model many common EAs, where crossover and cloning are often performed in some proportion to one another in the same generation. We have developed a framework that can address these algorithms relatively easily.

Figure 1 provides a model of a single generation during an 


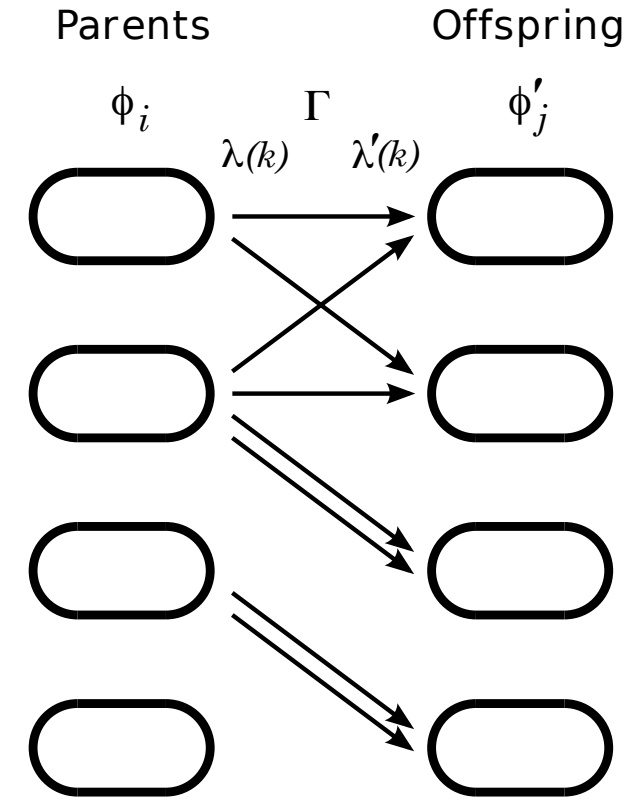

Figure 1: A sample generation.

EA run. Some parents are selected and produce offspring, either through crossover or cloning, with the potential for mutation being applied at some point during the process as well. Note that every relationship between parent and offspring is represented. This means that midparent calculations (averaging parent traits) is not appropriate for this model. Also note that cases where an offspring is produced by a single parent, instead of two, are represented using two links. It is important that each link represent the same amount of "influence" that a parent has on it's offspring. Thus all offspring have two arrows leading to them, even if they come from the same parent. If other operators are used that require more than two parents, the appropriate number of arrows will be the least common multiple of parents-peroffspring of all the operators involved.

A vector of phenotypic traits $\phi_{i}$ is associated with each parent $i$. Similarly a vector of traits $\phi_{j}^{\prime}$ is associated with each offspring $j$. The relationship between parents and offspring is defined by the by the set of links $\Gamma$, forming a directed graph. The two functions $\lambda(k)$ and $\lambda^{\prime}(k)$ are defined such that they return the appropriate parent index and offspring index respectively, for a given link $k$. This allows one to match up corresponding sets of parent and offspring traits, such as $\phi_{\lambda(k)}$ and $\phi_{\lambda^{\prime}(k)}^{\prime}$.

To abbreviate, we will sometimes refer to the traits of all parents as $\phi$ and all offspring as $\phi^{\prime}$. These actually indicate matrices where the row identifies an individual and the column identifies a specific trait. Similarly $\phi_{\lambda}$ refers to all traits of the selected parents, and $\phi_{\lambda^{\prime}}^{\prime}$ again refers to all the traits of the offspring, although in the case of figure 1 there are two copies of each child.

With the knowledge that $\operatorname{var}\left(\phi^{\prime}\right)=\operatorname{var}\left(\phi_{\lambda^{\prime}}^{\prime}\right)$, and the iden- tity $\operatorname{var}(a X+b Y)=a^{2} \operatorname{var}(X)+b^{2} \operatorname{var}(Y)+2 a b \operatorname{cov}(X, Y)$, we can derive the following equation by setting $X=\phi_{\lambda}$, $Y=\phi_{\lambda^{\prime}}^{\prime}, a=-1$, and $b=1$.

$$
\begin{aligned}
\operatorname{var}\left(\phi^{\prime}\right) & =\operatorname{var}\left(\phi_{\lambda^{\prime}}^{\prime}\right) \\
\operatorname{var}\left(\phi^{\prime}\right) & =2 \operatorname{cov}\left(\phi_{\lambda^{\prime}}^{\prime}, \phi_{\lambda}\right)+\operatorname{var}\left(\phi_{\lambda^{\prime}}^{\prime}-\phi_{\lambda}\right)-\operatorname{var}\left(\phi_{\lambda}\right) .
\end{aligned}
$$

If we define the matrices $\mathbf{O}=\operatorname{var}\left(\phi^{\prime}\right), \mathbf{G}^{\prime}=\operatorname{cov}\left(\phi_{\lambda^{\prime}}^{\prime}, \phi_{\lambda}\right)$, $\mathbf{D}=\operatorname{var}\left(\phi_{\lambda^{\prime}}^{\prime}-\phi_{\lambda}\right)$, and $\mathbf{P}=\operatorname{var}\left(\phi_{\lambda}\right)$, we can rewrite the equation as follows:

$$
\begin{aligned}
& \mathbf{O}=2 \mathbf{G}^{\prime}+\mathbf{D}-\mathbf{P} \\
& \mathbf{O}=\mathbf{P}\left[2 \mathbf{G}^{\prime} \mathbf{P}^{-1}+\mathbf{D} \mathbf{P}^{-1}-\mathbf{I}\right]
\end{aligned}
$$

where $\mathbf{I}$ is the identity matrix.

In equation 5, everything within the brackets defines a transformation matrix that transforms the distribution of the traits of the selected parents $(\mathbf{P})$ into the distribution of the offspring population traits $(\mathbf{O})$. The factor $\mathbf{G}^{\prime} \mathbf{P}^{-1}$ is very similar to the quantitative genetics notion of heritability. It is important to note that the $\mathbf{G}^{\prime}$ in these equations is not quite the same as the $\mathbf{G}$ in equation 3 . In fact, $\mathbf{G}=\tau \mathbf{G}^{\prime}$, as long as $\tau$ (the number of parents per offspring) is constant for every operation performed. In other words, as long as there is no mixing of crossover and cloning. This means that in the case of crossover for example, $\mathbf{G}^{\prime} \mathbf{P}^{-1}$ represents the degree to which the traits of a single parent have been inherited, rather than both parents.

Equation 5 also exposes the term $\mathbf{D P} \mathbf{P}^{-1}$, which is not commonly seen in quantitative genetics equations. We refer to this as perturbation, since it describes the amount of new variation that the operators are introducing into the population. It is our contention that perturbation measures an operator's capacity for exploration, while heritability measures it's potential for exploitation.

\subsection{Covariance Matrices Metrics}

When biologists consider the multivariate notion of heritability, they tend to think of it as the degree of similarity between the two probability distributions that $\mathbf{P}$ and $\mathbf{G}$ describe. These comparisons are often performed using statistical techniques like Common Principle Component Analysis $[7,8]$. In our experiments with CPCA, we found its output to be too course grained to be useful to us as a diagnostic tool, and so we have developed our own approach.

For simplicity and ease of understanding, we would like to find a metric that expresses terms like heritability and perturbation as a single scalar value. We have chosen to use the following metric,

$$
\mathrm{m}\left(\mathbf{G}^{\prime} \mathbf{P}^{-1}\right)=\sqrt[M]{\operatorname{det}\left(\mathbf{G}^{\prime} \mathbf{P}^{-1}\right)}
$$

where $\mathrm{m}$ is the metric function, and $\mathbf{G}^{\prime}$ and $\mathbf{P}$ are $M$ by $M$ covariance matrices as described in the previous section. $M$, in other words, indicates the number of traits being measured.

The result of equation 6 is, of course, our scalar version of heritability from a single parent. Similarly, $\sqrt[M]{\operatorname{det}\left(\mathbf{D P}^{-1}\right)}$ would measure perturbation, and $\sqrt[M]{\operatorname{det}\left(\mathbf{O P}^{-1}\right)}$ gives us a 
measure of the overall similarity between the selected parent population and the resulting offspring population.

We chose to use determinants because they have an intuitive geometric interpretation. They are equal to the volume of a parallelogram that has its dimensions defined by the eigenvectors of the matrix. Thus the determinant gives us a rough measure of the volume under the curve of the distribution that the covariance matrix represents. Determinants do have one disadvantage though. They will evaluate to a zero if any of the traits in the populations becomes fixed (i.e. converged). This is why all the algorithms we will examine contain at least a small amount of mutation. We also use Schäfer and Strimmer's [21] shrinkage approach for estimating covariance matrices, that is guaranteed to produce semi-positive definite matrices.

We take the $M^{\text {th }}$ root of each determinant in order to modify the units of the measure to be in line with those of a single trait. This makes interpretation more intuitive, much the way standard deviation values are often easier to interpret than variances.

We should note that this similarity metric could be potentially misleading in some situations. It is possible for two matrices that are fairly different to still have the same determinant. On the other hand, though, whenever the determinants are different, there is no way the matrices could be the same.

\section{OBSERVING A SIMPLE EA}

Before we begin looking at the Pittsburgh approach operators, we would like to set a baseline so that we know what we should expect to see, and thus what we would like to achieve with our customized operators. It also gives us a chance to compare the results of these measures against our existing understanding of how an EA works.

\subsection{Standard Gaussian Mutation}

We began by examining standard Gaussian mutation with a fixed $\sigma$ (standard deviation) on a very simple function optimization problem. Specifically, we chose what is often referred to as the sphere function. The goal is to minimize the following fitness function,

$$
f(x)=\sum x^{2}
$$

where $x$ is a vector of 10 real-valued genes. There are no explicit bounds placed on the gene values, but when the population is initialized, genes are randomly chosen in the range $[-5.12,5.12)$. The Gaussian mutation is applied to every gene during reproduction, with $\sigma=0.2$ remaining constant throughout the run.

Choosing quantitative traits for this problem is fairly straightforward. Each of the real-valued parameters to the fitness function make an ideal trait, especially since we tend to visualize the landscape in terms of this space. Since there is a direct relationship between the genes and the parameters, each gene value becomes one of our traits.

In figure 2, we plot the behavior of our EA on the sphere function, averaged over 30 runs. In all cases where we do this averaging, the averaged lines are reasonable representations of individual runs. We have plotted lines for $\sqrt[M]{\operatorname{det}\left(\mathbf{G}^{\prime} \mathbf{P}^{-1}\right)}$, $\sqrt[M]{\operatorname{det}\left(\mathbf{D P}^{-1}\right)}$ and $\sqrt[M]{\operatorname{det}\left(\mathbf{O P}^{-1}\right)}$. These allow us to examine the heritability, perturbation, and overall change in the population respectively. We have also plotted the line $\sqrt[10]{\operatorname{det}(\mathbf{P})}$, signifying the overall size of the $\mathbf{P}$ matrix. This allows us to see how much the population has converged.

As we can see, the heritability of the mutation operator remains fairly high throughout the run, dipping only briefly just as the population is beginning to converge. This remained consistent over a wide range of values for $\sigma$. The perturbation, on the other hand, begins quite low, and then rapidly increases just as the population convergence begins to slow dramatically. This is exactly what we would expect to see when using a fixed Gaussian mutation with a fixed $\sigma$. As the population converges, it will eventually reach a point where the distribution of the population is similar in scale to the distribution generated by the Gaussian mutation. At that point it will become much more difficult, if not impossible, for the population to converge any further. The search process switches over to something much closer to random search, albeit in a very localized portion of the search space.

\subsection{Standard 2-Point Crossover}

Next we wanted to examine the behavior of a standard 2point crossover operator. Ideally we might like to run an EA with crossover alone, but we found that these techniques do not function well if there is too little variance in the population. Consequently, we maintained a small amount of Gaussian mutation with $\sigma=0.02$, which is applied after selection, but before crossover is applied. The probes that we use to measure the traits do not measure the effect of the mutation though. The traits of the individuals are examined directly before and after crossover is performed. In the following experiment, a crossover rate of 1.0 was used.

Figure 3 shows the effects of the 2-point crossover operator, averaged over 30 runs. The heritability from a single parent $\left(\mathrm{m}\left(\mathbf{G}^{\prime} \mathbf{P}^{-1}\right)\right)$ hovers close to 0.5 throughout the run, which is ideal for an operator that takes two parents. It may be somewhat hard to see on the plot, but both the perturbation $\left(\mathrm{m}\left(\mathbf{D} \mathbf{P}^{-1}\right)\right)$ and the overall population similarity $\left(\mathrm{m}\left(\mathbf{O P}^{-1}\right)\right)$ hover close to 1.0 throughout the run. Compared to figure 2, the high values for perturbation are impressive, and they do not come at any cost to the overall population similarity. This is to be expected, of course. It is well understood that crossover is a much more aggressive search operator, and that it has an adaptive quality that allows it to maintain it's search even as the population converges. Of course, if we were to try this on a landscape that contained epistasis, we would expect lower values for $\mathrm{m}\left(\mathbf{G}^{\prime} \mathbf{P}^{-1}\right)$.

This gives us a sense for how the prototypical versions of these operators work. As we examine customized operators, any deviations from these patterns will give us a sense for what the problem is, and a more detailed examination of the traits should allow us to zero-in on the specific issues involved.

\section{DIAGNOSING AN OPERATOR}




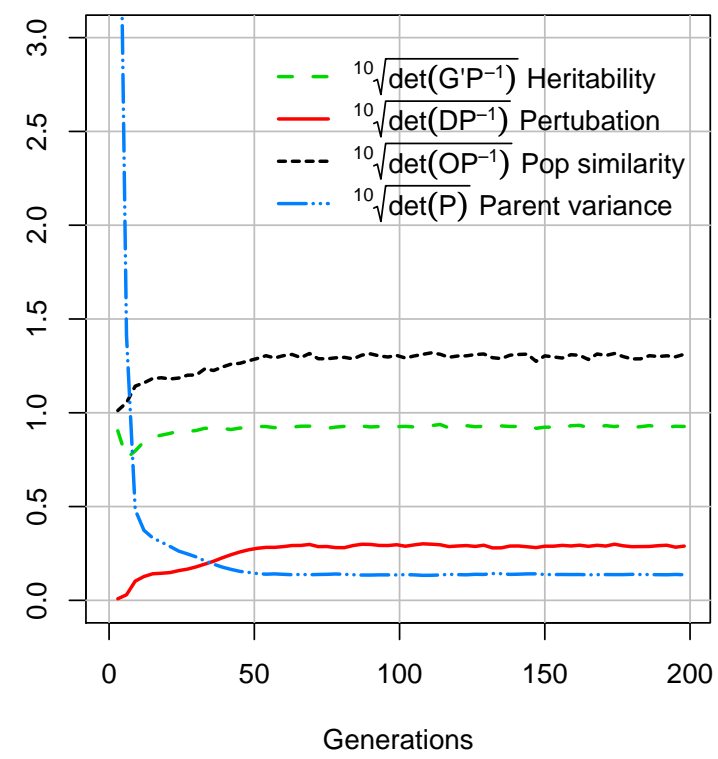

Figure 2: Gaussian mutation on the sphere function. A plot of $\mathbf{m}\left(\mathrm{G}^{\prime} \mathbf{P}^{-1}\right), \mathbf{m}\left(\mathrm{DP}^{-1}\right), \mathbf{m}\left(\mathrm{OP}^{-1}\right)$ and $\sqrt[10]{\operatorname{det}(\mathbf{P})}$, averaged over 30 runs.

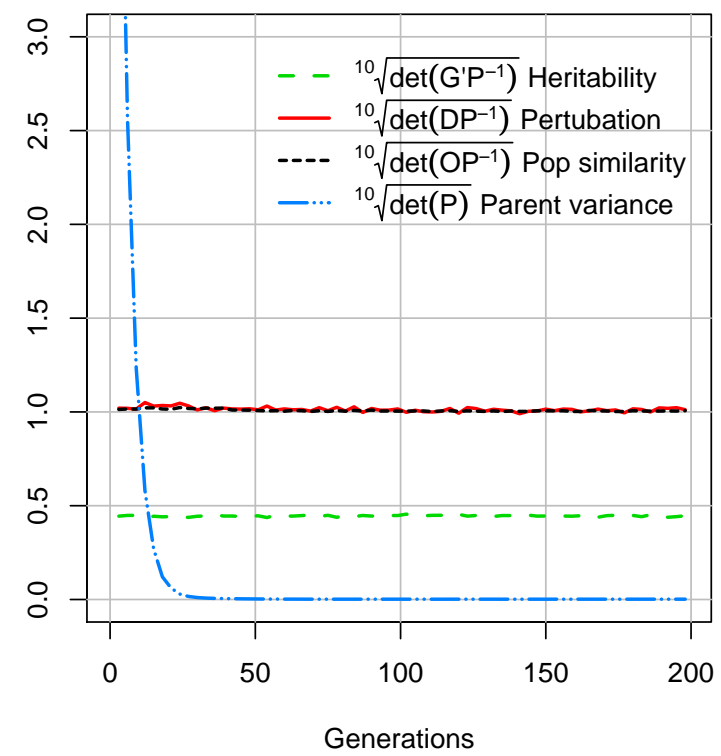

Figure 3: Standard 2-point crossover on the sphere function. A plot of $\mathbf{m}\left(\mathbf{G}^{\prime} \mathbf{P}^{-1}\right), \mathbf{m}\left(\mathbf{D P} \mathbf{P}^{-1}\right), \mathbf{m}\left(\mathbf{O P} \mathbf{P}^{-1}\right)$ and $\sqrt[10]{\operatorname{det}(\mathbf{P})}$, averaged over 30 runs. Gaussian mutation was also used, but it's effects are not measured here.
Here we present a demonstration of how quantitative genetics theory can be used to diagnose problems with one of the standard recombination operators for Pittsburgh approach classifier systems.

\subsection{Pittsburgh Approach}

Learning classifier systems are a machine learning technique that use evolutionary algorithms to evolve sets of rules. The distinguishing characteristics of the Pittsburgh approach are that each individual defines an entire rule-set, and the number or rules can vary from individual to individual. Because of the way rule-sets are interpreted, the position of the genes (rules) on the genome does not affect the semantics. In most systems rules are defined to be fixed length, with a constant number of conditions on the left-hand-side and a constant number of actions on the right-hand-side of each rule.

To support inductive learning, some sort of generalization mechanism is required. A number of different approaches have been used, including wildcards (a.k.a. "don't care" symbols), ranges, and nearest neighbor matching mechanisms that fire the rule whose conditions are the shortest distance from the input in condition space. The experiments we perform here will use something akin to the nearest neighbor approach.

For a variable length genome to be useful, its size must be able to adapt to the problem. The standard reproductive operators will not modify the genome size, so a custom recombination operator was developed. Instead of choosing cut points that are in the same place in both genomes, the cut points on each genome are chosen independently of one another. The result is that one offspring may get more genes from its parents than the other does.

This recombination operator has never worked as well as hoped. Numerous attempts have been made to find better replacements. The most successful approach has been the development of a homologous recombination operator that compared genes on the two genomes, and only swapped genes that were similar. Unfortunately the matching process could become quite expensive for large genomes.

\subsection{Function Approximation}

We chose a problem domain that allowed us to create a test problem that is simple enough to demonstrate the diagnosis process, is well as creating a more complex problem to properly test our operators to see how they perform. The function approximation domain also made it fairly simple to define real-valued phenotypic traits using the sampling method described above.

\subsubsection{Representation}

The genome of an individual is a string of concatenated rules. All rules have the same length, but each genome can have a different number of rules. The rules consists of a single condition/input and a single action/output. Thus a rule identifies a single point on a function $g(x)=y$, where $x$ is the input and $y$ is the output.

The generalization mechanism is similar to nearest neighbor, but instead of returning the output of the the rule with the 
Table 1: Points defining the target function

\begin{tabular}{|r|r||r|r|}
\hline $\mathrm{X}$ & $\mathrm{Y}$ & $\mathrm{X}$ & $\mathrm{Y}$ \\
\hline-10.00 & -7.0 & 3.62 & -10.0 \\
\hline-3.80 & -9.0 & 5.11 & 7.0 \\
\hline-2.22 & -6.0 & 6.48 & -5.0 \\
\hline-2.15 & -1.0 & 8.61 & 2.0 \\
\hline 2.75 & 8.0 & 10.00 & 9.0 \\
\hline
\end{tabular}

closest condition, an interpolation is returned between the two rules who's conditions bound it on either side. When an input value does not lie between two rules, the output value of the one closest rule is returned. The result is that each individual defines a piecewise-linear function, where the rules define each the vertices.

When individuals are randomly initialized in the first generation, all input and output values lie within the range $[-10,10]$. There is nothing in place to enforce this range past initialization though.

\subsubsection{Target Functions}

While it is possible to approximate almost any function using this representation, we chose a function that could be represented exactly by an ideal individual. We were concerned that a smooth function might encourage the EA to learn very large rule-sets in order to get the best approximations possible. This might complicate our analysis.

Our target function was defined using the points shown in Table 1. The function that these points describe is plotted in Figure 4. A very simple function seemed appropriate for the diagnosis.

For performing rigorous tests of the operators, a more complex test function was developed. It consists of 50 randomly generated points within the same $[-10,10]$ range.

\subsubsection{Fitness}

Fitness is calculated using a set of 30 training examples that are drawn randomly from the target function. A new training set is drawn each generation with the goal of reducing over-fitting. The fitness function is defined as follows,

$$
f(X)=\frac{1}{N} \sum_{i=1}^{N}\left[g\left(X_{i}\right)-Y_{i}\right],
$$

where $X$ and $Y$ are vectors containing the training set inputs and corresponding outputs, $N$ is the number of training examples (30 in this case), and $g(x)$ is the piecewise-linear function as defined by the individual being evaluated.

An independent test set containing 30 examples is also generated at the beginning of a run. These play no role in the learning process, and are used solely to evaluate the learning process.

\subsection{Choosing Quantitative Traits}

When choosing quantitative traits for analyzing the operators, two goals should be kept in mind. First we would like our traits to be representative of the underlying phenotype, and second we want our traits to help lead us back to

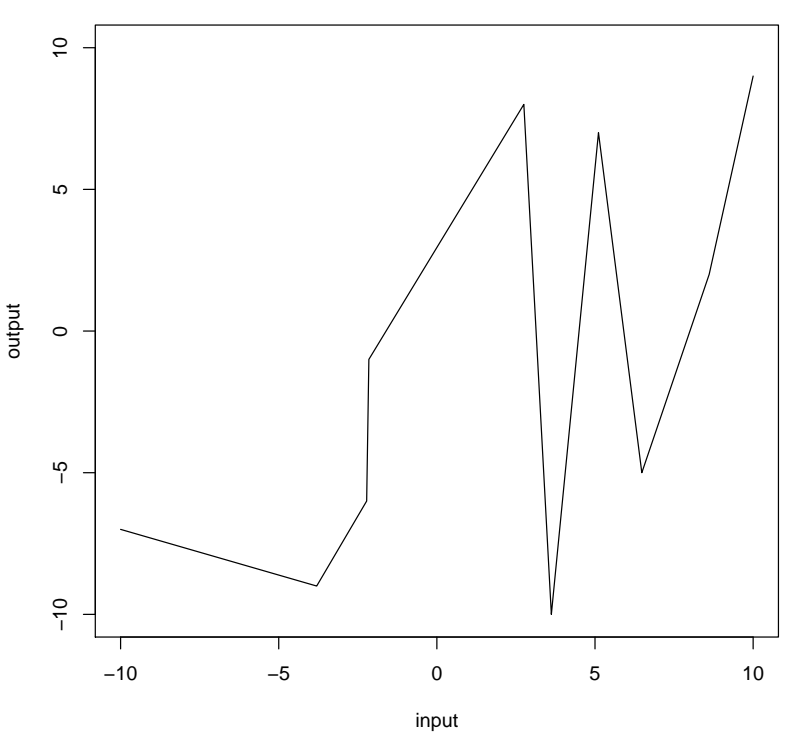

Figure 4: The target function to be learned.

where problems are occurring in the genetic structure of our individuals.

For a Pittsburgh approach system, we considered two possible views of the phenotype: high-level behaviors, and the input-output map. The high-level-behavior view is particularly appropriate when one is working with an agent based model or robotics domain. Certain behaviors that seem important for the specific domain can be quantified. Things such as obstacle avoidance, navigation, goal seeking and tracking could all be quantified, often in terms of the frequency or duration of certain model events or situations. The advantage to this approach is that, with careful thought, the traits are very likely to accurately and comprehensively describe the phenotype space. The disadvantage is that they could be far removed from the underlying representation, making it difficult still to diagnose the actual problem.

Another way to think about the phenotype is as a mapping between inputs and outputs. This approach has the advantage of being much closer to the representational structure, thus having the potential to identify problems more easily. The disadvantage is that quantifying the entire input/output map is nearly impossible for anything but the most trivial of problems. The only hope is to sample points in the space and hope that they can provide enough information to be useful. This approach is most easily applied to very simple problems.

Given both the ease of implementation and the likelihood of producing relevant information, we chose to use the second approach of sampling the input/output space. We selected a set of 10 sample inputs that will be passed to each individual. These inputs are evenly distributed across the condition/input space. The corresponding output at each sample point is a quantitative trait, giving us 10 traits total. This 
decision influenced our choice of test problems as well, as we will see in the next section.

\subsection{Looking Under the Hood}

To get an initial sense of the behavior of the Pittsburgh (Pitt) 2-point crossover operator, we performed an experiment on the function approximation problem described in table 1. A population of 100 individuals was used, and all individuals were initialized to contain 10 rules, the ideal number needed to solve the problem. Crossover was performed at a rate of 1.0 , and prior to individuals being passed to the crossover operator, Gaussian mutation is applied with a mutation rate of 1.0 and $\sigma=0.05$. As was the case with the standard 2-point crossover, the effects of mutation on the phenotypic traits are not measured.

The standard 2-point crossover operator cannot change the size of an individual, but the Pitt crossover can and does. In order to control bloat (constant genome growth), parsimony pressure is applied in the form of a fitness penalty when the Pitt operator is used. We used a penalty of 0.01 per rule. All plots that display fitness show the raw fitness values, before the penalty is applied.

Thirty runs were performed, and the average metrics are displayed in figure 6 . When compared with the metrics from the standard 2-point crossover in figure 3, we notice a couple of things. First, the perturbation curve is much higher than with the standard 2-point crossover, ranging from 1.5 at the beginning of the run, up to 2.0 by the end, indicating that this operator may be over-extending it's search. Perhaps more importantly though, the heritability curve is quite low, averaging around 0.3 . This indicates that an EA using this operator may have problems converging past a certain point, limiting its ability to find good solutions.

While helpful, this analysis does not give us quite enough information to diagnose any issues with the crossover operator. Next we will analyze the relationship between the various traits to see if we can get a better understanding of what is causing the low heritability.

\subsection{Identifying the Problem}

Aggregated information about the traits, and the relationships between them, is all stored within the matrices. We thought that by carefully examining them, the source of our problem might become clear. We are particularly concerned with the heritability of the operator, so we chose to examine the $\mathbf{G}^{\prime} \mathbf{P}^{-1}$ matrix. We begin by examining the $\mathbf{G}^{\prime} \mathbf{P}^{-1}$ matrix for the standard 2-point crossover operator from the experiment shown in figure 3 .

For each generation we calculated the 30 heritability matrices from each run, and then averaged them together, producing one matrix per generation. Below we provide part of the average matrix from generation 5 , as an example. Note that the variances along the diagonal tend to have values near 0.5 , while all the off-diagonal covariances are fairly negligible.

$$
\left[\begin{array}{rrrrrr}
0.523 & -0.005 & -0.005 & -0.003 & & -0.008 \\
-0.005 & 0.550 & -0.010 & -0.007 & \cdots & -0.007 \\
-0.005 & -0.010 & 0.552 & -0.010 & & -0.007 \\
-0.003 & -0.007 & -0.010 & 0.520 & & -0.004 \\
& \vdots & & & \ddots & \\
-0.008 & -0.007 & -0.007 & -0.004 & & 0.542
\end{array}\right]
$$

When we compare this with the $\mathbf{G}^{\prime} \mathbf{P}^{-1}$ matrices associated with the Pittsburgh 2-point crossover, we noticed something interesting. In the early generations of the run, certain pairs of traits had much higher covariances than others. Based on this, we deduced that those traits were linked in some way. We provide a part of the average matrix from generation 5 below, as an example.

$$
\left[\begin{array}{rrrrrr}
0.528 & -\mathbf{0 . 1 2 2} & -0.014 & -0.003 & & -0.002 \\
-\mathbf{0 . 1 2 2} & 0.470 & -\mathbf{0 . 0 4 1} & -0.003 & \cdots & -0.005 \\
-0.014 & \mathbf{- 0 . 0 4 1} & 0.392 & \mathbf{- 0 . 0 2 9} & & -0.004 \\
-0.003 & -0.003 & -\mathbf{0 . 0 2 9} & 0.391 & & -0.007 \\
& \vdots & & & \ddots & \\
-0.002 & -0.005 & -0.004 & -0.007 & & 0.420
\end{array}\right]
$$

Each trait is represented by both a row and a column in a covariance matrix, with row $i$ and column $i$ representing the the same trait. The numbers along the diagonal (shown in italics) are the variances of the each trait, and the other numbers are covariances between two different traits identified by the specific row and column. In the above example, the numbers displayed in bold indicate the unusually high values.

Our traits were all measured using probes at consecutive input values. This means that the high covariance values all appear to be between neighboring traits. Since we chose traits that would be closely related to the genes in our representation, we can infer that rules that have similar conditions tend to have higher linkages between them. A crossover operator that can reduce the frequency of cuts between highly linked genes should have a higher heritability.

\subsection{An Improved Recombination Operator}

Taking what we have learned into consideration, we decided to create what we call the "condition space crossover operator". Instead of performing cuts on the genome explicitly, our crossover performs cuts in condition space.

In the function approximation domain we have only one condition per rule. Our new operator first sorts the rules of both parents by the condition value. This sorting is not only done within each parent, but the rules of both parents are sorted relative to one another as well (see figure 5 for an illustration). This creates $l_{1}+l_{2}-1$ possible cut points, where $l_{1}$ and $l_{2}$ are the lengths of the two parents. Two cut points $c_{1}$ and $c_{2}$ are then selected, and the rules falling between $c_{1}$ and $c_{2}$ in each parent are exchanged, creating the two offspring.

Figure 7 shows the metrics for an experiment run using the 


\begin{tabular}{ll|l|l|l|l|} 
Parent 1: & Rule & Rule & Rule & Rule & \\
Parent 2: & Rule & Rule & Rule & Rule & Rule \\
& &
\end{tabular}

Child 1: Rule Rule Rule Rule Rule

Child 2: Rule Rule Rule Rule Rule

Figure 5: An illustration of the Condition Space Crossover operator. Rules are sorted by condition value both within and between parents. Crossover points are then selected such that only rules with similar condition values are exchanged between parents.

new crossover operator. As before, 30 runs were performed with a population size of 100 , a crossover rate of 1.0 , Gaussian mutation $\sigma=0.05$ and a parsimony penalty of 0.01 . As we can see, the single parent heritability has increased to an average of close to 0.4 throughout the run. In addition, the perturbation has decreased somewhat, especially early in the run, and leveled off roughly near 1.5. The population of selected parents has also converged more rapidly, and on a smaller volume than with the Pitt crossover operator.

Figure 10 shows a comparison of the single parent heritability measures $\left(\mathrm{m}\left(\mathbf{G}^{\prime} \mathbf{P}^{-1}\right)\right)$ of the two operators. Whiskers are plotted showing the confidence intervals of these curves. The condition space crossover has a significantly higher heritability than the Pitt 2-point operator.

To give the operators a proper test, we used the 50 point test function described in section 5.2.2. We compared both operators using EAs that both had population size of 100 , Gaussian mutation with $\sigma=0.01$, parsimony penalty $=$ 0.01 , and individuals initialized with 50 rules each, instead of the normal 10. The best-so-far training results (Figure 8) and testing results (Figure 9) show that our new operator significantly outperforms the Pitt 2-point crossover. The increase in heritability and decrease in perturbation seems to have provided a more appropriate balance for achieving an effective search.

\section{CONCLUSIONS}

We have demonstrated that multivariate quantitative genetics theory can provide a framework for practitioners to answer questions about their algorithms, even when specific theories do not yet exist. Much of the effort in using this approach will be expended on developing an appropriate set of quantitative traits. For the practitioner though, this is a much less daunting task than developing new theory to address their problems. And as we saw in our example, even a subset of traits can yield the information necessary to make a diagnosis.

There are a number of steps we are taking at this point. With this successful proof of principle on rule representations/operators, we are now applying this approach to a second difficult representation/operator issue, finite state automata, to show its generality. We are also developing

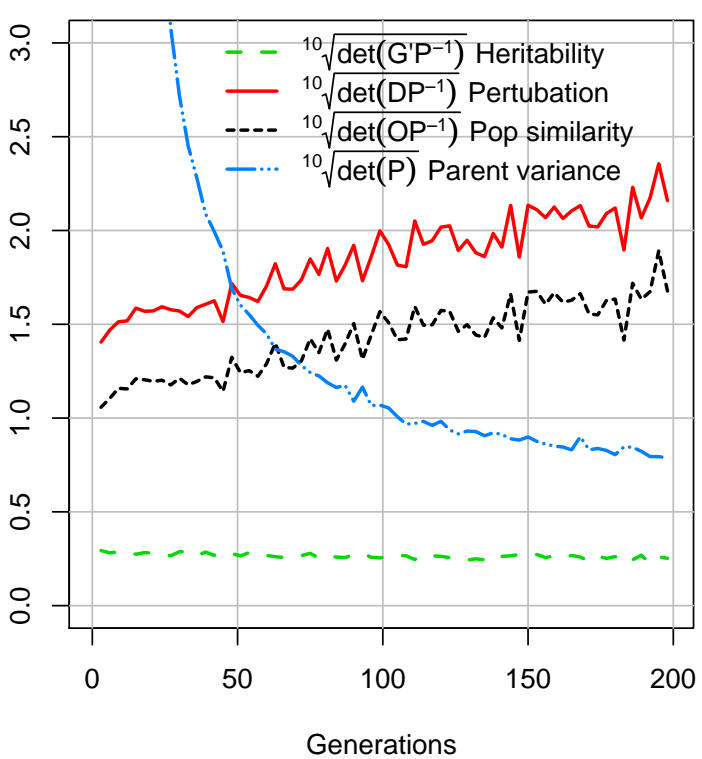

Figure 6: Pitt 2-point crossover on the function approximation problem shown in figure 4 . A plot of $\mathbf{m}\left(\mathbf{G}^{\prime} \mathbf{P}^{-1}\right), \mathbf{m}\left(\mathbf{D P} \mathbf{P}^{-1}\right), \mathbf{m}\left(\mathbf{O P}^{-1}\right)$ and $\sqrt[10]{\operatorname{det}(\mathbf{P})}$, averaged over 30 runs. Gaussian mutation was also used, but it's effects are not measured here.

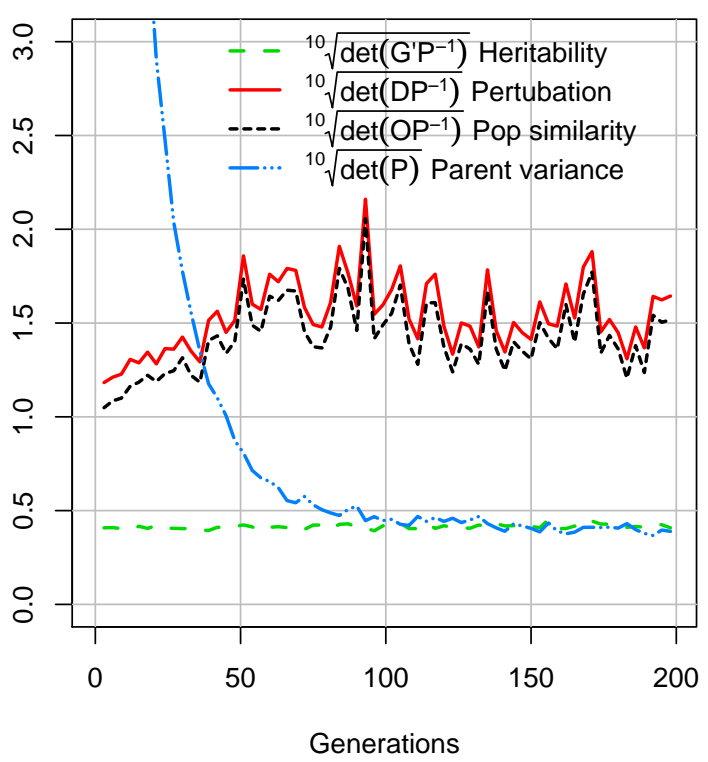

Figure 7: Condition space crossover on the function approximation problem shown in figure 4. A plot of $\mathbf{m}\left(\mathbf{G}^{\prime} \mathbf{P}^{-1}\right), \mathbf{m}\left(\mathbf{D P} \mathbf{P}^{-1}\right), \mathbf{m}\left(\mathbf{O P}^{-1}\right)$ and $\sqrt[10]{\operatorname{det}(\mathbf{P})}$, Gaussian mutation was also used, but it's effects are not measured here. 


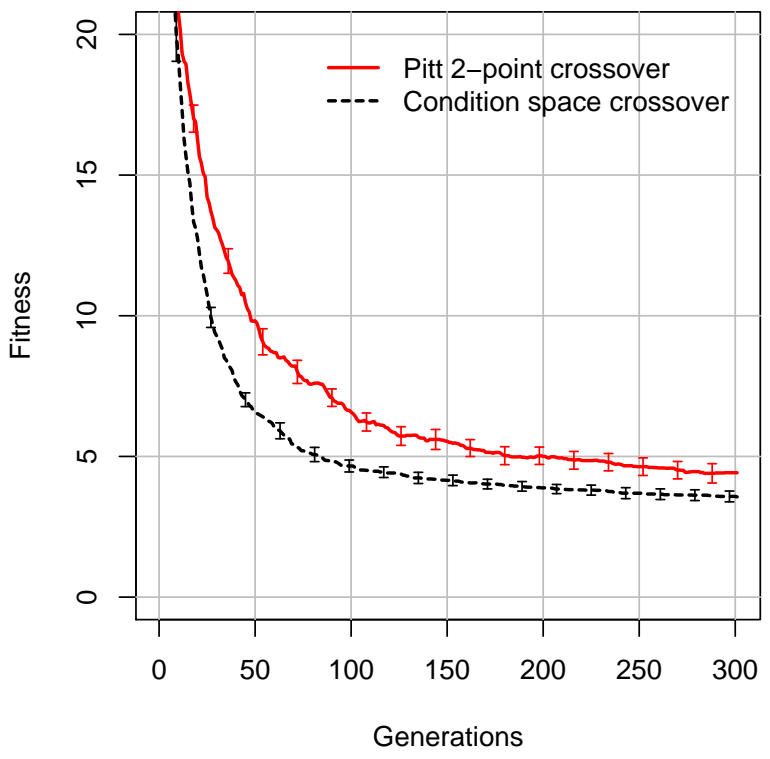

Figure 8: Average Best-so-far plot of training results.

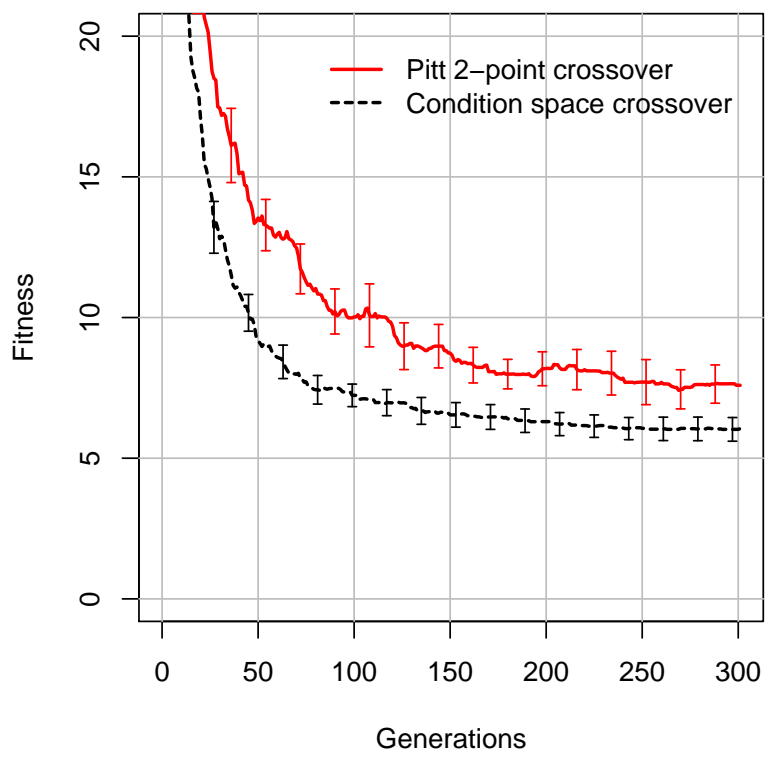

Figure 9: Plot of average test set results on best-sofar.

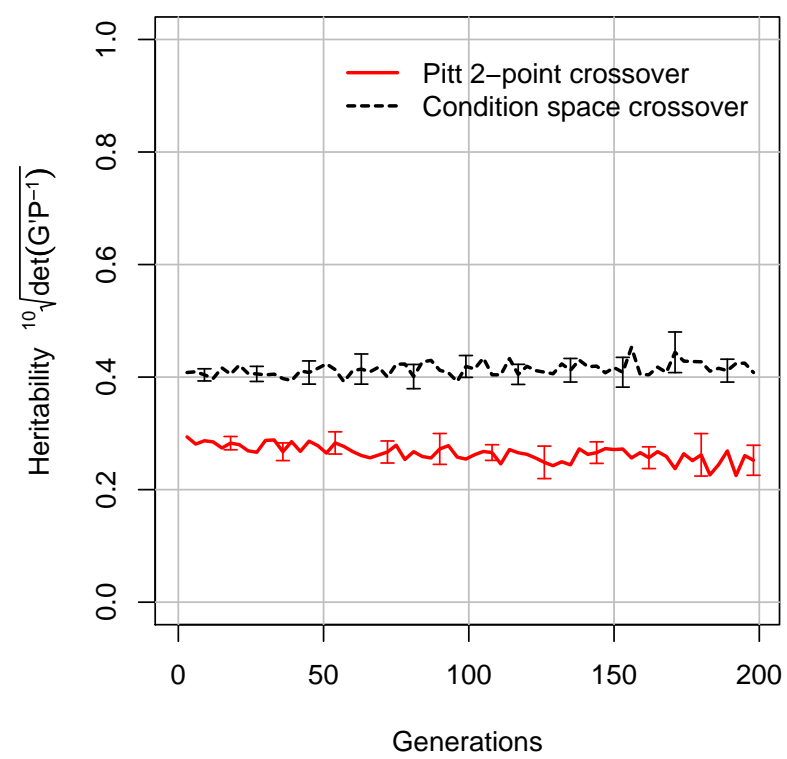

Figure 10: A comparison of the heritability of both Pitt approach crossover operators.

a covariance matrix similarity metric that addresses some of the short-comings of the one presented here.

\section{REFERENCES}

[1] L. Altenberg. The schema theorem and Price's theorem. In L. D. Whitley and M. D. Vose, editors, Foundations of Genetic Algorithms III, pages 23-49, San Francisco, CA, 1995. Morgan Kaufmann.

[2] S. J. Arnold. Multivariate inheritance and evolution: a review of concepts. Quantitative Genetic Studies of Behavioral Evolution, pages 17-48, 1994.

[3] H. Asoh and H. Mühlenbein. Estimating the heritability by decomposing the genetic variance. In Y. Davidor, H.-P. Schwefel, and R. Männer, editors, Parallel Problem Solving from Nature - PPSN III, pages 98-107, Berlin, 1994. Springer. Lecture Notes in Computer Science 866.

[4] J. K. Bassett, M. A. Potter, and K. A. De Jong. Looking under the EA hood with Price's equation. In K. Deb, R. Poli, W. Banzhaf, H.-G. Beyer, E. Burke, P. Darwen, D. Dasgupta, D. Floreano, J. Foster, M. Harman, O. Holland, P. L. Lanzi, L. Spector, A. Tettamanzi, D. Thierens, and A. Tyrrell, editors, Genetic and Evolutionary Computation GECCO-2004, Part I, volume 3102 of Lecture Notes in Computer Science, pages 914-922, Seattle, WA, USA, 26-30 June 2004. Springer-Verlag.

[5] J. K. Bassett, M. A. Potter, and K. A. De Jong. Applying Price's equation to survival selection. In H.-G. Beyer, U.-M. O'Reilly, D. V. Arnold, W. Banzhaf, C. Blum, E. W. Bonabeau, E. Cantu-Paz, D. Dasgupta, K. Deb, J. A. Foster, E. D. de Jong, 
H. Lipson, X. Llora, S. Mancoridis, M. Pelikan, G. R. Raidl, T. Soule, A. M. Tyrrell, J.-P. Watson, and E. Zitzler, editors, GECCO 2005: Proceedings of the 2005 Conference on Genetic and Evolutionary Computation, volume 2, pages 1371-1378, Washington DC, USA, 25-29 June 2005. ACM Press.

[6] D. S. Falconer and T. F. C. Mackay. Introduction to quantitative genetics. Longman New York, 1981.

[7] B. Flury. Common Principal Components and Related Multivariate Models. Wiley series in probability and mathematical statistics. Wiley, New York, 1988.

[8] E. T. Game and M. J. Caley. The stability of P in coral reef fishes. Evolution, 60(4):814-823, 2006.

[9] T. Jones and S. Forrest. Fitness distance correlation as a measure of problem difficulty for genetic algorithms. In L. Eshelman, editor, Proc. of the Sixth Int. Conf. on Genetic Algorithms, pages 184-192, San Francisco, CA, 1995. Morgan Kaufmann.

[10] R. Lande. Quantitative genetic analysis of multivariate evolution, applied to brain: Body size allometry. Evolution, 33(1):402-416, Mar. 1979.

[11] R. Lande and S. J. Arnold. The measurement of selection on correlated characters. Evolution, 37(6):1210-1226, Nov. 1983.

[12] W. B. Langdon. Genetic Programming and Data Structures: Genetic Programming + Data Structures = Automatic Programming! The Kluwer international series in engineering and computer science. Kluwer Academic Publishers, Boston, 1998.

[13] B. Manderick, M. de Weger, and P. Spiessens. The genetic algorithm and the structure of the fitness landscape. In R. K. Belew and L. B. Booker, editors, Proc. of the Fourth Int. Conf. on Genetic Algorithms, pages 143-150, San Mateo, CA, 1991. Morgan Kaufmann.

[14] H. Mühlenbein, J. Bendisch, and H.-M. Voigt. From recombination of genes to the estimation of distributions: II. continuous parameters. In H.-M. Voigt, W. Ebeling, I. Rechenberg, and H.-P. Schwefel, editors, Parallel Problem Solving from Nature - PPSN $I V$, pages 188-197, Berlin, 1996. Springer.

[15] H. Mühlenbein and G. Paaß. From recombination of genes to the estimation of distributions: I. Binary parameters. In H.-M. Voigt, W. Ebeling, I. Rechenberg, and H.-P. Schwefel, editors, Parallel Problem Solving from Nature - PPSN IV, pages 178-187, Berlin, 1996. Springer.

[16] H. Mühlenbein and D. Schlierkamp-Voosen. Predictive models for the breeder genetic algorithm: I. continuous parameter optimization. Evolutionary Computation, 1(1):25-49, 1993.

[17] M. A. Potter, J. K. Bassett, and K. A. De Jong. Visualizing evolvability with Price's equation. In R. Sarker, R. Reynolds, H. Abbass, K. C. Tan, B. McKay, D. Essam, and T. Gedeon, editors, Proceedings of the 2003 Congress on Evolutionary Computation CEC2003, pages 2785-2790, Canberra, 8-12 Dec. 2003. IEEE Press.

[18] G. Price. Fisher's 'fundamental theorem' made clear. Annals of Human Genetics, 36(2):129-140, 1972.

[19] G. R. Price. Selection and covariance. Nature, 227:520-521, Aug. 1970.
[20] S. H. Rice. Evolutionary Theory: Mathematical and Conceptual Foundations. Sinauer Associates, Inc., 2004.

[21] J. Schäfer and K. Strimmer. A shrinkage approach to large-scale covariance matrix estimation and implications for functional genomics. Statistical Applications in Genetics and Molecular Biology, 4(1):1175, 2005.

[22] P. F. Stadler. Correlation in landscapes of combinatorial optimization problems. Europhys. Lett., 20:479-482, 1992.

[23] E. Weinberger. Correlated and uncorrelated fitness landscapes and how to tell the difference. Biological Cybernetics, 63(5):325-336, 1990.

[24] B. Yuan and M. Gallagher. Experimental results for the special session on real-parameter optimization at CEC 2005: a simple, continuous EDA. In D. Corne, Z. Michalewicz, B. McKay, G. Eiben, D. Fogel, C. Fonseca, G. Greenwood, G. Raidl, K. C. Tan, and A. Zalzala, editors, Proceedings of the 2005 IEEE Congress on Evolutionary Computation, volume 2, pages 1792-1799, Edinburgh, Scotland, UK, 2-5 Sept. 2005. IEEE Press.

[25] B. Yuan and M. Gallagher. A mathematical modelling technique for the analysis of the dynamics of a simple continuous EDA. In G. G. Yen, S. M. Lucas, G. Fogel, G. Kendall, R. Salomon, B.-T. Zhang, C. A. C. Coello, and T. P. Runarsson, editors, Proceedings of the 2006 IEEE Congress on Evolutionary Computation, pages 1585-1591, Vancouver, BC, Canada, 16-21 July 2006. IEEE Press. 